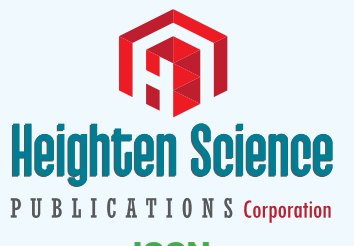

ISSN

2573-7724
*Address for Correspondence: Ryosuke Kohno, Medical Physics Section, National Institute of Radiological Sciences, 4-9-1, Anagawa, Inage, 263-8555, Chiba, Japan,

Email:kohno.ryosuke@qst.go.jp

Submitted: 04 February 2019

Approved: 13 February 2019

Published: 14 February 2019

Copyright: @ 2019 Kohno R, et al. This is an open access article distributed under the Creative Commons Attribution License, which permits unrestricted use, distribution, and reproduction in any medium, provided the original work is properly cited

Keywords: Variable RBE; Intensity-modulated proton therapy (IMPT); Monte carlo

Check for updates
Research Article

\section{Biological dose calculation using variable RBE in Single- and Multi- field Optimization IMPT plans for 3 Brain Tumor patients}

\author{
Ryosuke Kohno ${ }^{1-3 *}$, Wenhua $\mathrm{Cao}^{2}$, Pablo Yepes ${ }^{2}$, Xuemin $\mathrm{Bai}^{2}$, \\ Falk Poenisch ${ }^{2}$, David R Grosshans ${ }^{2}$, Tetsuo Akimoto ${ }^{3}$ and \\ Radhe Mohan ${ }^{4}$
}

${ }^{1}$ Medical Physics Section, National Institute of Radiological Sciences, 4-9-1, Anagawa, Inage, 263-8555, Chiba, Japan

2Department of Radiation Physics, Unit 94, The University of Texas MD Anderson Cancer Center, Houston, TX 77030, USA

${ }^{3}$ Division of Radiation Oncology and Particle Therapy, National Cancer Center, Kashiwa, 2778577, Chiba, Japan

${ }^{4}$ Department of Radiation Oncology, Unit 1150, The University of Texas MD Anderson Cancer Center, Houston, TX 77030, USA

\section{Abstract}

Purpose: The purpose of this study was to evaluate biological dose in single-field optimization (SFO) and multi-field optimization (MFO) intensity-modulated proton therapy (IMPT) plans for brain tumor patients that used a fixed relative biological effectiveness (FRBE) and those that used a variable RBE (VRBE).

Materials and methods: SFO and MFO IMPT plans were planned by the Varian Eclipse treatment planning system for three brain tumor patients. Dose and linear energy transfer (LET) distributions for each plan were recomputed using an in-house fast Monte Carlo dose calculator system, and then biological dose distributions were calculated with a FRBE of 1.1 or with a previously published VRBE model. We then compared biological dose distributions obtained by the VRBE with those obtained by the FRBE.

Results: Doses obtained by the VRBE for the gross tumor volume and clinical target volume in all plans were $1 \%-2 \%$ larger than those obtained by the FRBE. The minimum dose obtained by the VRBE for the brainstem in the SFO IMPT of one patient was $140 \%$ larger than that obtained by the FRBE, but the difference was only $5.3 \mathrm{cGy}$ (RBE). The difference in maximum dose for the optic chiasm in the MFO IMPT of another patient was less than $3.2 \%$, but the dose difference was $149.2 \mathrm{cGy}$ (RBE). We also found that no major differences were seen between the biological dose differences in the SFO IMPT plans and those in the MFO IMPT plans.

Conclusion: We could observe biological dose differences between the FRBE and the VRBE in the SFO and the MFO IMPT plans for brain tumor patients.

\section{Introduction}

In current proton beam therapy, clinical dose is conventionally prescribed by scaling the physical proton dose using a proton relative biological effectiveness (RBE) relative to photons of 1.1. However, the RBE of protons depends on the linear energy transfer (LET), the dose per fraction, and the tissue type [1]. Since the RBE varies, the assumption that the RBE is a constant value of 1.1-that is, a fixed RBE (FRBE) - may limit the potential of proton beam therapy. Wedenberg et al., evaluated the impact of 
disregarding variations in RBE in the comparison of proton and photon treatment plans [2], and Paganetti and Chen et al., discussed the potential clinical impact of a variable RBE (VRBE) [3,4].

The latest proton beam scanning technique [5-7], can deliver dose more conformally than passive scattering proton beam therapy and can meet the clinical constraints of using state-of-the-art intensity-modulated proton therapy (IMPT) [8]. Clinical impact of a VRBE calculation for the IMPT has been evaluated by Frese et al. [9]. Currently, Kohno et al., reported biological dose comparisons between a RBE and a VRBE in SFO and MFO IMPT for a brain tumor [10]. For the clinical target volume, biological doses obtained by the VRBE were 1\%-2\% larger than those obtained by the FRBE. On the other hand, for organs at risk (OARs) located in the end of the beam, biological dose with the conventional FRBE had any dose errors in IMPT plans.

Here, since they evaluated only in one case, it is not sufficient to support their conclusion. Therefore, in this paper, we investigated biological dose comparison between the FRBE and the VRBE obtained in the SFO and MFO IMPT plans for three brain tumor patients with various tumor volume and tumor's location.

\section{Materials and Methods}

We selected three brain tumor patients, who were treated with proton therapy at the University of Texas MD Anderson Cancer Center. Figure 1 shows the target volumes, target locations and organs at risk in the brain tumor treatment plans on a computed tomography slice. The tumors are surrounded in a complicated manner by nerve, brain, brainstem, bone, and sinus cavity. The gross tumor volume (GTV) and the clinical target volume (CTV) for each case are $6.3 \mathrm{cc}$ and $111.8 \mathrm{cc}$ for case (A), $37.4 \mathrm{cc}$ and $70.4 \mathrm{cc}$ for case (B), and $27.1 \mathrm{cc}$ and $84.4 \mathrm{cc}$ for case (C).

SFO and MFO IMPT plans were designed by the Eclipse treatment planning system (version 13.5; Varian Medical Systems, Inc., Palo Alto, CA). We used the same three coplanar beams in both IMPT plans for each patient. The prescribed proton radiotherapy doses were 5700 and 5000 cGy (RBE) to the GTV and CTV, respectively, in 30 fractions. Using a minimum dose and a maximum dose objective function with the same optimization conditions for both IMPT plans, all plans were designed to cover $100 \%$ of the GTV and the CTV and to minimize the maximum dose for the brainstem, the optic chiasma, both optic nerves, which the maximum dose constraint is 5400 cGy (RBE).

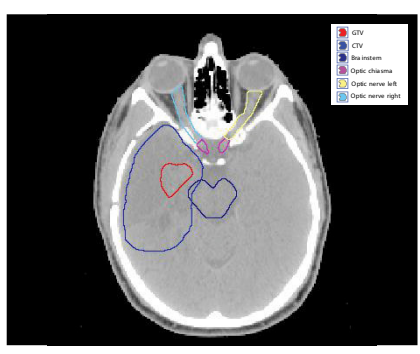

(A)

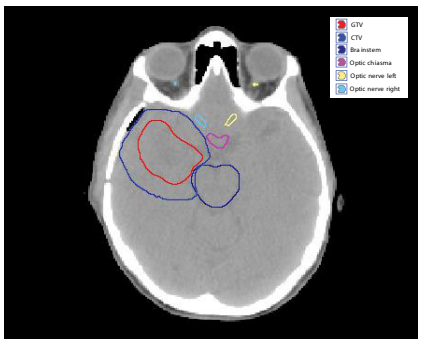

(C)

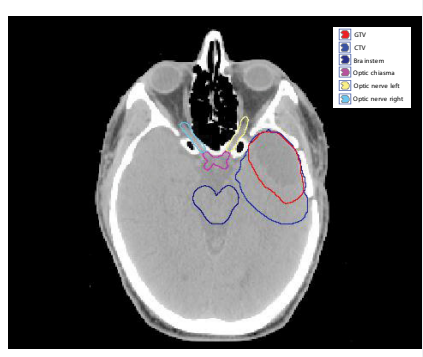

(B)

Figure 1: Contours of the GTV, CTV and OARs drawn on the computed tomography image for three brain cancer patients. 
Physical dose and LET distributions for each plan were recomputed using an inhouse "fast dose calculator" (FDC) [11,12]. The FDC is a fast Monte Carlo method, and can calculate dose distributions in less than 5 minutes per patient and is suitable for routine clinical work. The FDC has been validated by Geant4 for IMPT plans [13] and compared with Eclipse treatment planning system [14].

Biological dose distributions were calculated using the FDC with an FRBE of 1.1 and using the VRBE model published by Wilkens et al. [15]. Using a reference radiation with parameters $a_{x}$ and $\beta_{x}$ based on the linear-quadratic (LQ) model, the RBE at proton dose $D_{p}$ on the dose averaged LET $L E T_{d}$ is given by

$$
\operatorname{RBE}\left(D_{p}, L E T_{d}, \alpha_{0}, \lambda, \alpha_{x}, \beta_{x}\right)=\frac{\sqrt{\alpha_{x}^{2}+4 \beta_{x} D_{p}\left(\alpha_{0}+\lambda L E T_{d}+\beta_{x} D_{p}\right)}-\alpha_{x}}{2 \beta_{x} D_{p}}
$$

where $a_{0}$ and $\lambda$ are the linear and the initial parameter, respectively, in terms of biologic response, $a$, to protons. We can describe the RBE as simply a function of the dose, the LET, and tissue-specific parameters. Biological parameters given by Frese et al. [9], were used in this study. To compare the FRBE and VRBE models, we performed a comparative analysis of dose distributions for each plan in each region of interest. The comparative analysis used the dosimetric parameters of minimum, maximum, and mean dose.

\section{Results and Discussion}

Figure 2 exemplifies iso-dose distributions of the FRBE(a) and the VRBE(b) in the SFO IMPT plan, and the FRBE(c) and the VRBE(d) in the MFO IMPT plan for a patient in figure 1(A). We could not observe visually differences among them. Figure 3 and 4 show dose volume histograms (DVH) of the target volumes such as the GTV and the $\mathrm{CTV}$, and the organs at risk (OAR) such as the brainstem, the optic chiasma, the left optic nerve and the right optic nerve in the SFO IMPT plans for each patient. DVHs in the MFO IMPT plans are shown in figures 5 and 6. Then, Figures 7 and 8 show dose averaged LET $L E T_{d}$ volume histograms in the SFO and MFO IMPT for each patient.

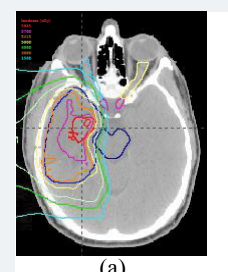

(a)

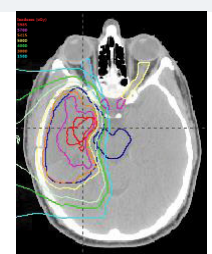

(b)

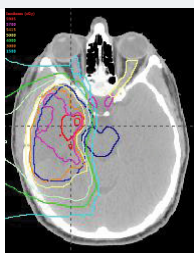

(c)

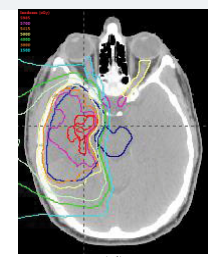

(d)

Figure 2: Iso-dose distributions of FRBE( a) and VRBE (b) for SFO IMPT plan, and FRBE (c) and VRBE (d) for MFO IMPT plan for patient of Figure 1(A).
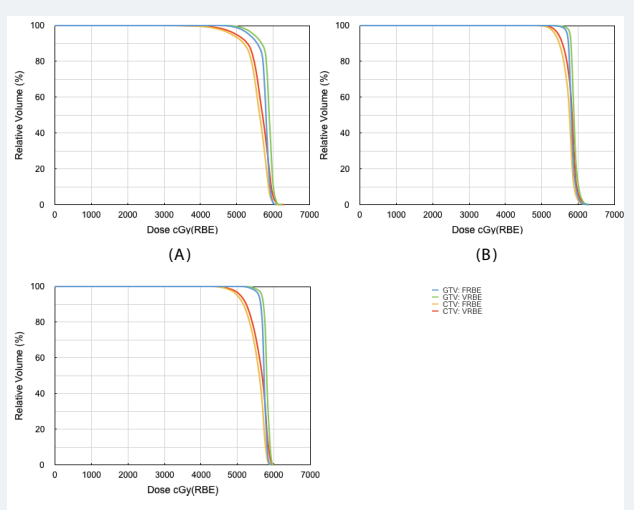

Figure 3: DVHs of GTV and CTV for FRBE and VRBE in SFO IMPT plans for each patient. 


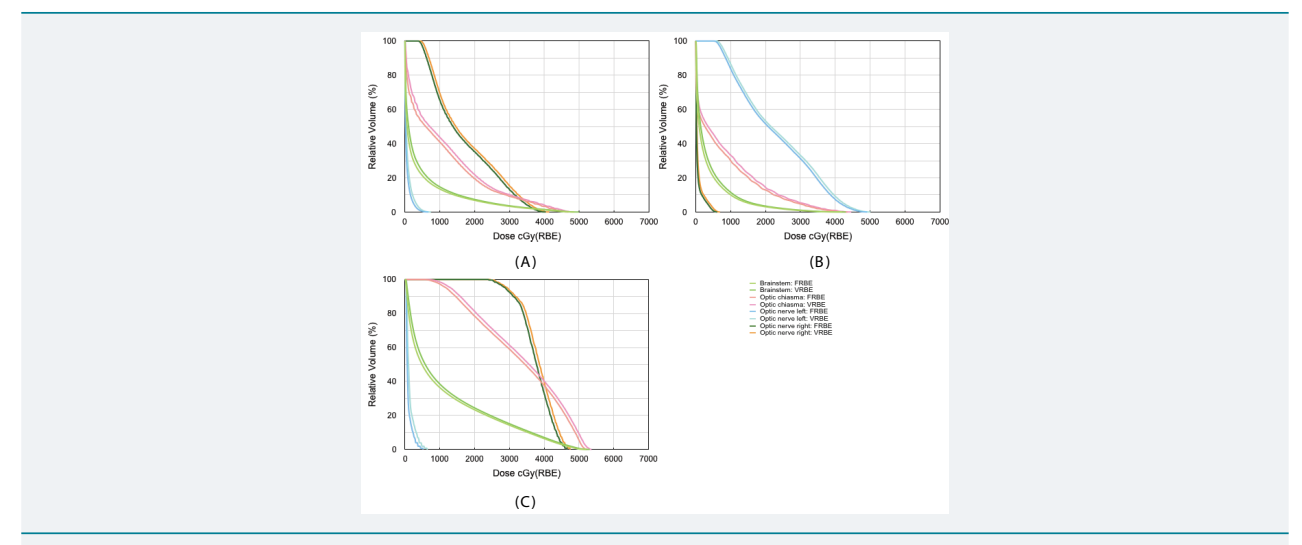

Figure 4: DVHs of brainstem, optic chiasma and both optic nerves for FRBE and VRBE in SFO IMPT plans for each patient.

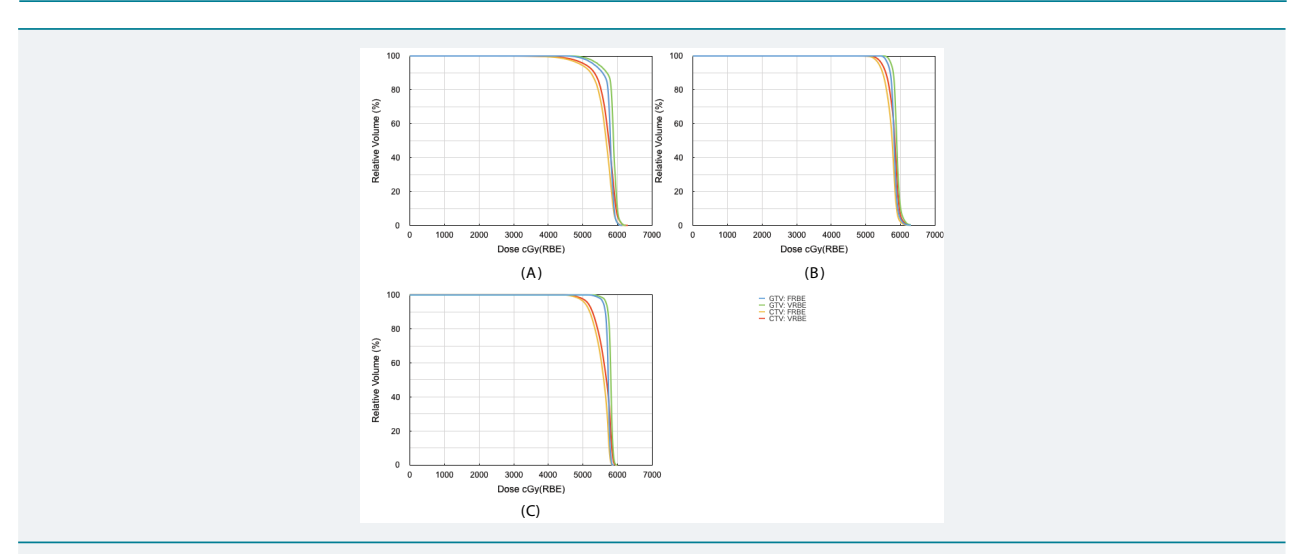

Figure 5: DVHs of GTV and CTV for FRBE and VRBE in MFO IMPT plans for each patient.
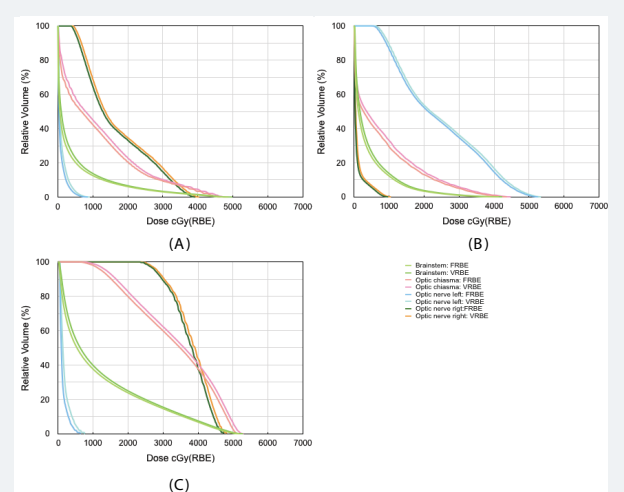

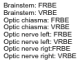

Figure 6: DVHs of brainstem, optic chiasma and both optic nerves for FRBE and VRBE in MFO IMPT plans for each patient.
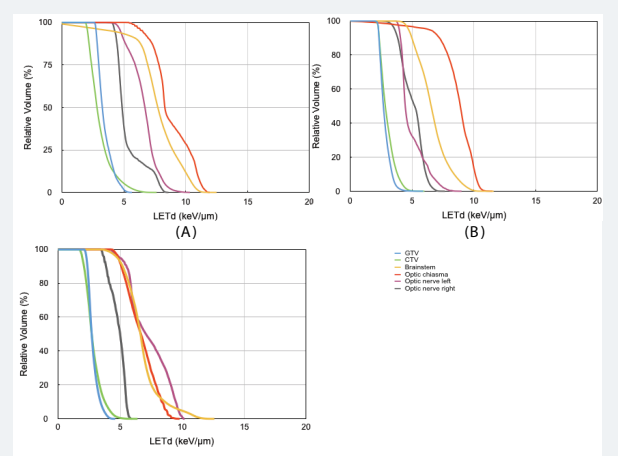

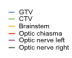

Figure 7: $L E T_{d}$ volume histograms of GTV, CTV, brainstem, optic chiasma and both optic nerves for FRBE and VRBE in SFO IMPT plans for each patient. 

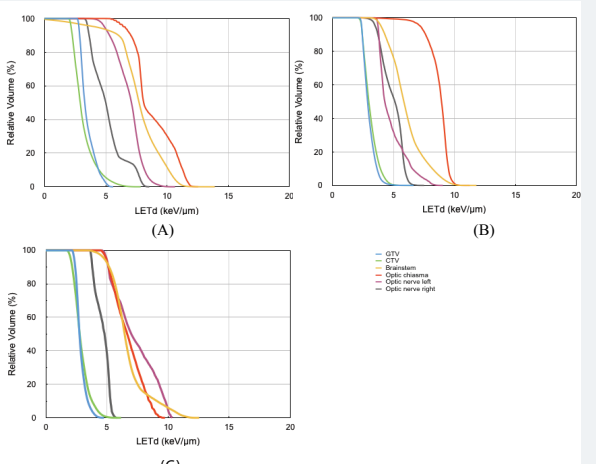

(B)

Figure 8: LET volume histograms of GTV, CTV, brainstem, optic chiasma and both optic nerves for FRBE and VRBE in MFO IMPT plans for each patient.

For the GTV, the SFO IMPT plans for three patients obtained by the FRBE and by the VRBE models differed by a mean ( \pm standard deviation) of $1.7 \pm 0.4 \%$ in minimum dose, $1.5 \pm 0.4 \%$ in maximum dose and $1.4 \pm 0.2 \%$ in mean dose. Those differences for the MFO IMPT plans were $2.6 \pm 0.9 \%, 1.7 \pm 0.2 \%$ and $1.5 \pm 0.2 \%$. For the CTV, the SFO IMPT plans differed by $2.7 \pm 1.8 \%$ in minimum dose, $1.5 \pm 0.4 \%$ in maximum dose and $1.4 \pm 0.1 \%$ in mean dose. Those differences for the MFO IMPT plans were $2.7 \pm 1.8 \%, 1.8 \pm 0.3 \%$ and $1.4 \pm 0.1 \%$. Overall, doses obtained by the VRBE for the target volumes were about $2 \%$ larger than those obtained by the FRBE as well as the results in the previous study [10].

For each OAR, the differences in minimum dose were considerably larger than those in maximum and mean dose. For the brainstem in the SFO IMPT of the patient (C), the minimum dose obtained by the VRBE was $139.5 \%$ larger than that obtained by the FRBE, and this was the largest difference due to the maximum $L E T_{d}$ of $12.6 \mathrm{keV} /$ $\mu \mathrm{m}$. However, the dose difference was only $5.3 \mathrm{cGy}$ (RBE). Additionally, the differences in maximum and mean dose were $2.0 \%$ but $103.4 \mathrm{cGy}$ (RBE), and $6.9 \%$ but $79.7 \mathrm{cGy}$ (RBE). In the SFO IMPT of the patient (A) with a target on the right side, the differences in minimum dose between the VRBE and the FRBE for the left optic nerve were $54.9 \%$ but only 2.8 cGy (RBE). The differences in maximum and mean dose were $9.9 \%$ but 66.7 cGy (RBE), and $41.3 \%$ but 23.8 cGy (RBE). In the SFO IMPT of patient (B) with a target on the left side, the differences in minimum dose between the VRBE and the FRBE for the right optic nerve was $73.9 \%$ but only 1.7 cGy (RBE). The differences in maximum dose and mean dose were $12.7 \%$ but 78.4 cGy (RBE), and $31.2 \%$ but 18.6 cGy (RBE).

The maximum dose to these critical organs is the most important clinical parameter. For the optic chiasm in the MFO IMPT of patient (A), the maximum dose obtained by the FRBE was 4641.4 cGy(RBE), and one obtained by VRBE was 4790.6 cGy(RBE). Namely, the difference in maximum dose in the optic chiasm was less than $3.2 \%$ but constituted the maximum dose difference of $149.2 \mathrm{cGy}$ (RBE). This maximum dose was much less than the maximum dose constraint of 5400 cGy (RBE).

Thus, we observed that doses obtained by the VRBE for OARs were larger than those obtained by the conventional FRBE, and these dose differences depended on location of OARs. Incidentally, we also confirmed that a one-to-one correlation coefficient $\mathrm{R}^{2}$ for each dose difference between the SFO plans and the MFO IMPT plans was 0.9883, and there were no differences between both plans. We also found that no major differences for $L E T_{d}$ volume histograms were seen between the SFO IMPT plans and the MFO IMPT plans for each patient. Namely, we did not observe any influence of the irradiation method.

It was obvious that biological dose calculation with the VRBE gave any dose differences in clinical case. These differences may be able to explain more precisely 
clinical effects. Naturally, biological dose distributions depend on the RBE calculation model. Although the phenomenologic RBE model proposed by Wilkens et al. [15], was used in this study, various RBE models have already been developed [16-19]. However, Patel et al., reported their RBE models show increasing discrepancy for higher $L E T$ values [20]. Since these cellular responses to proton beam irradiation have not been yet clearly modeled, it is necessary to improve the accuracy of existing RBE models or develop new models.

\section{Conclusion}

We evaluated biological dose differences between the conventional FRBE and the VRBE in the SFO and the MFO IMPT plans for three brain tumor patients. Doses obtained by the VRBE model for GTV and CTV were about $2 \%$ larger than those obtained by the FRBE model. On the other hand, doses obtained by the VRBE for OARs were somewhat larger than those obtained by the FRBE, and their dose differences depended on location of OARs. We also found that the differences in minimum dose for each OAR were larger than the differences in maximum and mean dose.

\section{Acknowledgments}

We thank Sarah Bronson from the Department of Scientific Publications at MD Anderson for editing the manuscript.

\section{Conflicts of interest}

The research described in this paper was supported by grant number U19 CA021239 from the National Cancer Institute, and Cancer Prevention and Research Institute of Texas (RP160232). The University of Texas MD Anderson Cancer Center is supported in part by the National Institutes of Health/National Cancer Institute through Cancer Center Support Grant P30CA016672.

\section{References}

1. Paganetti H. Relative biological effectiveness (RBE) values for proton beam therapy. Variations as a function of biological endpoint, dose, and linear energy transfer. Phys Med Biol. 2014; 59: R419-R472. Ref.: https://goo.gl/Yto1xP

2. Wedenberg $M$, Toma-Dasu I. Disregarding RBE variation in treatment plan comparison may lead to bias in favor of proton plans. Med Phys. 2014; 41: 091706-9. Ref.: https://goo.gl/WrdexA

3. Paganetti $\mathrm{H}$. Relating proton treatments to photon treatments via the relative biological effectivenessshould we revise current clinical practice? Int J Radiat Oncol Biol Phys. 2015; 91: 892-894. Ref.: https://goo.gl/zr4E8F

4. Chen $\mathrm{Y}$, Grassberger $\mathrm{C}$, Li J, Hong TS, Paganetti $\mathrm{H}$. Impact of potentially variable RBE in liver proton therapy. Phys Med Biol. 2018; 63: 195001. Ref.: https://goo.gl/BqtTS3

5. Pedroni E, Bacher R, Blattmann H, Bohringer T, Coray A, et al. The 200-MeV proton therapy project at the Paul Scherrer Institute: conceptual design and practical realization. Med Phys. 1995; 22: 37-53. Ref.: https://goo.gl/LvJ1cm

6. Gillin MT, Sahoo N, Bues M, Ciangaru G, Poenisch F, et al. Commissioning of the discrete spot scanning proton beam delivery system at The University of Texas M.D. Anderson Cancer Center, Proton Therapy Center, Houston. Med Phys. 2010; 37: 154-163. Ref.: https://goo.gl/kPYDA7

7. Kohno R, Hotta K, Dohmae T, Matsuzaki T, Nishio T, et al. Development of continuous line scanning system prototype for proton beam therapy. Int J Particle Ther. 4: 429-438. Ref.: https://goo.gl/mNkXqx

8. Kooy HM, Grassberger C. Intensity modulated proton therapy. Br J Radiol. 2015; 88: 20150195 Ref.: https://goo.gl/74xAjC

9. Frese MC, Wilkens JJ, Huber PE, Jensen AD, Oelfke $U$, et al. Application of constant vs. variable relative biological effectiveness in treatment planning of intensity-modulated proton therapy. Int $J$ Radiat Oncol Biol Phys. 2011; 79: 80-88. Ref.: https://goo.gl/Uzu8hS

10. Kohno R, Cao W, Yepes P, Bai X, Poenisch F, et al. Biological dose comparison between a Fixed RBE and a variable RBE in SFO and MFO IMPT with various multi-beams for brain cancer. IJMPCERO. 2019; 8: 32-45. Ref.: https://goo.gl/N8DyRx 
11. Yepes $P$, Randeniya S, Taddei PJ, Newhauser WD. A track repeating algorithm for fast Monte Carlo dose calculations of proton radiotherapy. Nucl Technol. 2009; 168: 736-740. Ref.: https://goo.gl/8MvBd5

12. Yepes P, Randeniya S, Taddei P J, Newhauser WD. Monte Carlo fast dose calculator for proton radiotherapy: application to a voxelized geometry representing a patient with prostate cancer. Phys Med Biol. 2009; 54: N21-N28. Ref.: https://goo.gl/FkxQb4

13. Yepes $P$, Eley JG, Liu A, Mirkovic D, Randeniya S, et al. Validation of a track repeating algorithm for intensity modulated proton therapy: clinical cases study. Phys Med Biol 2016; 61: 2633-2645. Ref.: https://goo.gl/Xf7qDy

14. Kohno R, Cao W, Yepes P, Bai X, Poenisch F, et al. Dose Comparison between Eclipse Dose Calculation and Fast Dose Calculator in Single- and Multi-Field Optimization Intensity-Modulated Proton Therapy Plans with Various Multi-Beams for Brain Cancer. IJMPCERO 2017; 6: 421-432. Ref.: https://goo.gl/sijxvg

15. Wilkens JJ, Oelfke U. A phenomenological model for the relative biological effectiveness in therapeutic proton beams. Phys Med Biol. 2004; 49: 2811-2825. Ref.: https://goo.gl/swW13K

16. Carabe-Fernandez A, Dale RG, Jones B. The incorporation of the concept of minimum RBE (RbEmin) into the linear-quadratic model and the potential for improved radiobiological analysis of high- LET treatments. Int J Radiat Biol. 2007; 83: 27-39. Ref.: https://goo.gl/vo2tzR

17. Carabe-Fernandez A, Dale RG, Hopewell JW, Jones B, Paganetti H. Fractionation effects in particle radiotherapy: implications for hypo-fractionation regimes. Phys Med Biol. 2010; 55: 5685-5700. Ref.: https://goo.gl/rXpM7w

18. Wedenberg $M$, Lind BK, Hardemark B. A model for the relative biological effectiveness of protons: the tissue specific parameter alpha/beta of photons is a predictor for the sensitivity to LET changes. Acta Oncol. 2013; 52: 580-588. Ref.: https://goo.gl/2cWJDs

19. McNamara AL, Schuemann J, Paganetti H. A phenomenological relative biological effectiveness (RBE) model for proton therapy based on all published in vitro cell survival data. Phys Med Biol. 2015; 60: 8399-8416. Ref.: https://goo.gl/xehj9s

20. Patel D, Bronk L, Guan F, Peeler CR, Brons S, et al. Optimization of Monte Carlo transport parameters and validation of a novel high throughput experimental setup to measure the biological effects of particle beams. Med Phys. 2017; 44: 6061-6073. Ref.: https://goo.gl/BxJcNh 